\title{
Some real-world applications of wireless sensor nodes
}

\author{
Steven D. Glaser ${ }^{1}$ \\ College of Engineering, University of California, 440 Davis Hall, Berkeley, CA, USA 94720-1710
}

\begin{abstract}
This paper presents two case histories of the use of wireless sensor Mote technologies. These are devices that incorporate communications, processing, sensors, sensor fusion, and power source into a package currently about two cubic inches in size - networked autonomous sensor nodes. The first case discussed is the November, 2001, instrumentation of a blastinduced liquefaction test in Tokachi Port, Japan. The second case discussed is the dense-pak ${ }^{\mathrm{TM}}$ instrumentation of the seismic shaking test of a full-scale wood-frame building on the UCB Richmond shake table. The utility of dense instumentation is shown, and how it allows location of damage globally unseen. A methodology of interpreting structural seismic respose by Bayesian updating and extended Kalman filtering is presented. It is shown that dense, inexpensive instrumentation is needed to identify structural damage and prognosticate future behavior. The case studies show that the current families of Motes are very useful, but the hardware still has difficulties in terms of reliability and consistancy. It is apparent that the TinyOS is a wonderful tool for computer science education but is not an industrual quality instrumentation system. These are, of course, growing pains of the first incarnations of the Berkeley Smart Dust ideal. We expect the dream of easy to use, inexpensive, smart, wireless, sensor networks to become a reality in the next couple of years.
\end{abstract}

Keywords: system identification, wireless instrumentation, structural response, MEMS sensors

\section{INTRODUCTION}

To date, much interest has been generated by a new family of wireless sensor nodes, i.e. Motes or smart dust. These are devices that incorporate communications, processing, sensors, sensor fusion, and power source into a package currently about two cubic inches in size - networked autonomous sensor nodes. They are designed to be embeddable throughout the environment - low cost (\$200 each now, $\$ 5$ each in the near future), non-obtrusive, unattended or unmanaged, and dynamically reprogrammable. The promise of the Mote is a self-organizing and adaptive information utility. Like the electric power utility, a Mote should be everywhere, always available, enabling virtually all of the tools of daily life, and be invisible to its users. TinyOS, an embedded operating system for Motes, allows multiple applications to be running while providing services such as messaging protocols, periodic timer events, static, persistent data storage, swapping of system components to create necessary functionality on the fly.

For the last three years we have worked towards bringing this vision to fruition. We have chosen to focus on mitigating problems associated with natural hazards. Vastly improved monitoring of structures is essential for providing rapid estimates of ground motion and widespread structural integrity after an earthquake. In this paper the term "structure" will be used in a very broad manner, using the definition "...anything built by people, from a dam built of earth or a pyramid of stone to a power station or an earth satellite" (Scott, 1993). For instance, the U.S. Geological Survey and the California Division of Mines and Geology have invested in strong motion instrumentation using conventional digital accelerometers. These installations have proven to be valuable sources of information, but they are limited in quantity because of high initial cost (on the order of $\$ 10,000$ each fully installed instrument) of sensors and significant operating costs.

1. glaser@ce.berkeley.edu; phone 1510 642-1264; fax 1 510642-1800; http://www.ce.berkeley.edu/ glaser/ 
We report on two full-scale field applications of Motes - what we tried to achieve, what went right, and what went wrong. The first case is the Tokachi Port, Japan, campaign where we installed twenty 2-D accelerometer Motes for the blastinduced liquefaction experiment in November, 2001. The second case study is the instrumentation of a full-size three story wood-frame building that underwent strong shaking on the UCB Richmond Field Station seismic shake table in December, 2001. These were the first field applications of Mote technology, and much was learned that has led to recent hardware and software improvements. We have worked directly with Professor Culler, author of TinyOS, and Prof. Pister, builder of smart dust, since the smart dust idea took form during the summer of 2001. Prof. David Culler beautifully describes the Berkeley Smart Dust ideal:.

Spread thousands of wireless sensor nodes casually over an arbitrary area of interest. They self-organize into a network conveying arbitrary information from any point to any other at whatever bandwidth is demanded... while operating at incredibly low energy usage (i.e, off most of the time) to run for years on small batteries and harvested energy...and being extremely responsive in times of key activity...without ever bothering you about design considerations, intended usage, faults, or constraints.

\section{THE MOTE}

\subsection{Hardware}

The Motes are simple, robust, and are designed to be built from readily available components. The basic structure of the original Mote baseboard (the "Rene") consists of an Atmel AT90LS2343-4SC microcontroller, and the RFMonolithics TR1000 amplitude-modulation 916.5 MHz Hybrid ASH Transceiver (Atwood et al., 2000). The devices were fitted with a Microchip Tech Inc. AT90LS2343-4SC IC serial EEPROM to act as non-volatile flash memory. The Rene boards were powered by two AA-sized alkaline batteries and did not have any on-board power control circuitry. The most common antenna was an $80 \mathrm{~mm}$ long copper wire. Some of the later Crossbow-packaged devices used a stubby $80 \mathrm{~mm}$ whip. At the time there was little direct optimization of hardware variables such as antenna design, micro-controller choice, or memory type. For instance, the Atmel micro-controller was chosen over the StrongArm and TI MSP430 because there was a gcc (free) compiler available through Gnu. We now see that the MSP430 is a much more powerful controller (16-

bit vs. 8-bit), uses less power than the Atmel, has better internal analog-to-digital convertors, has pre-implemented UARTs, etc. Dust Inc. and University of Twente currently make excellent use of the TI chip.

The detachable sensor board incorporated a two degree of freedom Analog Devices ADXL202e 2g MEMS accelerometer, and connected to the main board through a Hirose H-series 51-pin, $1 \mathrm{~mm}$ header. The Intel Laboratory at Berkeley, directed at the time by Prof. Culler, later tried to develop a five-sensor weather board. This board is currently being sold by Crossbow Inc. as the MTS420CA sensorboard. The weather board holds an ADXL202e accelerometer, the Sensirion SHT11 humidity and temperature sensor combination, the Taos TSL2550d light intensity sensor, and the Intersema MS5534AP pressure and temperature sensor combination.

\subsection{TinyOS (tiny operating system)}

A key innovative capability of the Mote is its pervasive support of fluid software, i.e., 1) it has the ability of processing, storage and data management functionality; 2) it can arbitrarily and automatically distribute itself among information devices and along paths through scalable computing platforms integrated with network infrastructure; 3 ) it can compose itself from preexisting hardware and software components; 4) it can satisfy its needs for services while advertising the services it can provide to others; 5) it can negotiate interfaces with service providers while adapting its own interfaces to meet components it serves. Recent progress in this area is the development of TinyOS, an embedded operating system for Motes. TinyOS migrates the event-based model being developed for clusters into a very light-weight form for Motes.

The heart of TinyOS (TOS, 2004), communications, must be able to scale to thousands or millions of nodes within a single network and be flexible to dynamic topology changes. It must also be tolerant of failures due to lossy links or 
failed nodes and support concurrency-intensive operations required by networked sensors with minimal hardware requirements. TinyOS implements the Active Messages model (Mainwaring and Culler, 1999; von Eicken et al., 1992) with intra- and inter-device connectivity to meet these requirements (Buonadonna and Hill, 2000) - Motes automatically detect the "best" routing topology and propagate all data to collection points (Woo and Culler, 2003). The TinyOS group has currently demonstrated the ability to deploy a self-configuring network of devices, but this work needs to be extended to support larger collections of devices. This will be done by improving networking protocols and by reducing the total amount of data being communicated back to a central location through automatic data aggregation inside the networks.

Because the Motes will be widely distributed, system power consumption must be limited, and software can play a major role in controlling the efficient use of the various hardware resources. This is particularly important in the case of the power-hungry radio components. Thus, future revisions of the software will focus on reducing power consumption as a primary goal while improving current levels of functionality.

\section{DENSE-PAK ${ }^{\text {TM }}$ STRUCTURAL HEALTH MONITORING}

The greatest public safety risk associated with natural hazards comes from the built environment. Knowledge of the integrity of structures can significantly reduce the risk of occupants and users by providing information about the collapse potential. To achieve this goal, we proposed to develop a dense-pak ${ }^{\mathrm{TM}}$ structural health monitor to enable buildings and bridges to monitor themselves with inexpensive Motes that locally sense and determine structural condition. Coupled with computational models residing in the Mote itself, and on-line through peer-to-peer networks, the monitoring system will obtain updated ground motion hazard estimates from reliable information sources, estimate damage and losses, and provide occupants and users with emergency information. The key to this approach are the sensor Motes - such systems must be inexpensive to install and maintain during original construction and after construction.

Following an earthquake, a structure will self-diagnose its ability to withstand loads and aftershocks in a safe manner, and provide occupants with customized information about the optimal emergency action through internaly and externaly distributed networks, PDA's, and alarms. The alarms will be integrated into a variety of other emergency scenarios (fire, medical, and security). Instead of the current time-consuming and chaotic manual procedure of inspectors tagging a building, waveforms monitored during the event will be compared in real time to numerical models and base-state measurements of safe building behavior, determining the building safety and integrity - notifying occupants of unsafe conditions in a process of self-tagging. Individual building monitor systems are linked through the wide-area network to distributed regional emergency response centers which prioritize the response of emergency personnel.

Local structural details may have significant effects on the whole system as well as the local responses, posing challenges for the interpretation of recorded system response after earthquake loading. From the collected data, evolution of system performance can be assessed, an essential task for performance-based engineering (Safak, 1997). A study of the Dumbarton Bridge, San Francisco Bay, by Fenves et al. (1992) emphasized the impact of articulation and longitudinal constraints at hinges on seismic response. Arici and Mosalam (2000) examined an instrumented portion of the Sylmar-I5/ I4 Interchange Bridge where accelerometers were placed on either side of an expansion joint designed to isolate span sections. During the first, smaller, earthquake, the two substructures of the bridge (separated by the expansion joint) responded in an independent manner. During the second, large, earthquake both sides of the joint behaved identically, implying that the joint did not behave as expected, i.e. the structure did not function as designed. Such specifics of the response can only be captured through dense-pak ${ }^{\mathrm{TM}}$ measurements.

The dense-pak ${ }^{\mathrm{TM}}$ system used for diagnosis of post-earthquake condition is in fact a "Smart Building" network to provide on-going information about structural condition and early warning of inadequate safety from a variety of causes. The wealth of data will lead to new economic opportunities for firms that provide analysis and evaluation for building owners based on dense, detailed, sensed information (e.g. Senera Inc.). This is particularly important in high-tech and biotech 
manufacturing facilities, data server farms, and other industrial and commercial buildings where valuable building contents are at risk because of excessive vibration or other movements.

\subsection{Structural System Identification and Evaluation}

By far the most common approach to structural damage prognoses has been the application of global modal analysis (e.g. McConnell, 1995). The method has shown some success, but it is now commonly accepted that modal analysis is far too insensitive to yield usable information for practical cases (Farrar et al., 2001). A prime example is the modal analysis work undertaken on the abandoned I-40 bridge across the Rio Grande river in Albuquerque, NM. It was only after the main longitudinal plate girder was cut more than $2 / 3$ through that any change was seen in the modal parameters. The first two modes dropped by a mere 7.6 and 4.4 percent respectively (Farrar and Doebling, 1997), which would be considered noise in a blind prediction case!

Global modal analysis is doomed for several reasons. Structures of interest are complex systems with a great number of degrees of freedom. Because evolving damage is local, a structure will redistribute internal forces to stiffer members as particular beams, columns, etc. are weakened. It is only when damage is sufficient to affect the performance of the entire structure that will it be visible through global modal analysis - well after the safety of the structure is exceeded.

We tried to implement a new approach to structural health prognosis, based on the evaluation of local damage, leveraging ubiquitous, cheap, wireless Motes, requiring a dense array of sensor nodes placed in swarms at key structural points throughout a structure, e.g. a dozen autonomous nodes, each carrying a 3-D accelerometer, distributed around a key beamcolumn connection. A structural engineer can identify zones in the structure where critical loading is expected. Using a variety of techniques (e.g. Stubbs and Park, 1996; DeHoop an DeHoop, 2000; Jain et al., 2001) we will optimize locations (as to extracting the maximum amount of information from the system) of the sensor clusters.

For the proposed scenario of perhaps thousands of nodes monitoring a large structure, it is not feasible to merely send back all the recorded signals from all the multi-sensored nodes to a common hub. Advances in information technology are key to realization of this health prognosis system, for several reasons.

A system of thousands of sensors would be hopelessly complex to address from a central server, require too much power from the wireless nodes, and would overwhelm the radio bandwidth. Tests on the current sensor node incarnation show that broadcasting 1 bit of information costs almost 11,000 times the power as local computation on a single bit.

Intelligent local arrays can monitor the evolution of local damage in real time, since the nodes function as a local network, able to evaluate data and make decisions (rather than merely collect data). The nodes can locally evaluate system changes amongst themselves, and "encode" the data by sharing decisions and evaluations rather than raw data.

Damage prognosis requires seamless integration of the measuring and modeling process, with constant updating of the model and information sensed.

\subsection{Approach to structural data interpretation}

Development of analytical tools to capture the evolution of system response in terms of damage initiation and damage propagation - understanding the interaction between the structural system and its components - is essential for performance-based design. The so-called system identification (SI) approach is a powerful and tidy statistical-based tool to quantify and assess system damage parameters, and has been so applied by many structural researchers (e.g., Udwadia, 1985; Beck, 1978; Gersch and Brotherton, 1982; Safak, 1988; Werner et. al., 1994; Arici and Mosalam, 2000; Baise and Glaser, 2000; Glaser and Baise, 2000).

System identification requires a model, whether black-box (e.g. a linear filter model) or white box (a physical model). Identification can be made through the extended Kalman filter (EKF), (Hoshiya and Saito, 1984; Lin and Zhang, 1994; Koh and See, 1994) which has been successfully applied to the identification of various physical systems. Physical 
parameters, including elastic moduli and damping coefficients, can be identified (Lin and Zhang, 1994; Beck and Katafygiostis, 1998; Smyth et al., 1998; Lus et al., 1999; Glaser and Baise, 2000). Integration of finite element modeling with system identification of boundary conditions has been successfully made at UC Berkeley (Arici and Mosalam, 2000).

Possibly the most promising parameterization of an evolving system is a unified methodology based on Bayesian/StateSpace identification and adaptive estimation (e.g. Shumway and Stauffer, 1982; Ching and Glaser, 2003). The Bayesian probabilistic approach has the following advantages: (1) probabilistic methods have the ability to model system disturbances, (2) system identification problems are usually ill-conditioned. The Bayesian approach can usually regularize ill-conditioned problems into linear ones, and (3) the Bayesian approach produces a posterior distribution, instead of a single estimation, hence it eliminates the risk of incorrect estimation and results in a robust estimation and control method.

Stochastic state-space models provide more flexibility in modeling noises, and Markov chain properties result in a more elegant formulation than with other categories of models, e.g. input-output models. Our approach completely extracts all useful information from data, i.e. input and output of a linear dynamic system, via the sufficient statistics, which are a conditional distribution of system states with respect to system responses. The computations of likelihood functions are simplified by introducing the Kalman filtering algorithm.

\section{CASE STUDIES}

\subsection{Tokachi Port}

In December of 2001, we participated with the Port and Airport Research Institute, Yokosuga, Japan, in a full-scale experiment using controlled blasting to induce lateral spreading. We were invited by Dr. Takahiro Sugano, Director of the Structural Dynamics Division, to see how the Crossbow Rene Mote would perform under these difficult conditions. Tokachi Port is on the East coast of Hokaido, with temperatures below freezing during November, 2001. During the experiment, a large section of ground was tsubjected to successive, large underground explosions that caused the ground to liquefy. We were asked to measure ground accelerations, and the accelerations of surcharge weights representing buildings. The sensors were placed in two zones of the experiment area: (1) the improved ground zone, including the boxes acting as surcharge weights, and (2) the liquefaction area behind the seismically resistant sheet pile wall (PARI study area). A photograph of the site is shown in Fig. 1.

A total of twenty sensor Motes were used in the two zones of the experiment area, with eight sensors distributed in the improved ground zone. Of these eight, four were placed immediately in front of the surcharge weights to measure ground

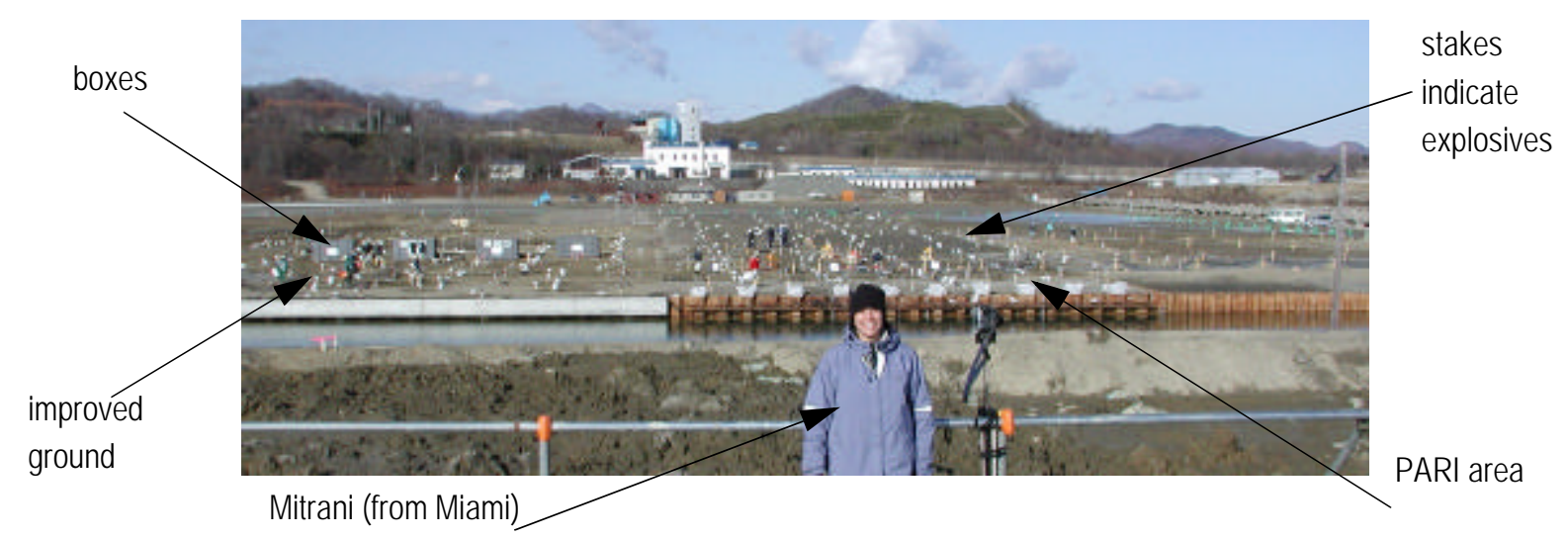

Figure 1. The Tokachi Port, Hokaido, site. 
accelerations. The other four sensors were placed on the side of the actual surcharge weights (boxes) to measure their accelerations relative to the ground. The remaining twelve nodes were distributed throughout the liquefaction area, placed behind the seismically resistant sheet pile wall to measure ground accelerations. Some of these nodes were placed alongside PARI's sensors for redundancy.

The reader should note that we were using the RFM 16.5 MHz amplitude modulation transceiver, and that the $900 \mathrm{MHz}$ band is restricted in Japan. Our crew, Judy Mitrani and Jan Goethals, observed that the Motes foundcommunications difficult to maintain during the day but that the devices seemed to work well in the evening. We thought the problem could be due to the batteries getting cold at night and warming during the day. As it were, the $900 \mathrm{MHz}$ frequency band is reserved for DoCoMo handy phones, and the RFMs were receiving constant cell phone traffic as well as their designated traffic. The RFM is not a particularly narrow band device, so the spurious signals were not rejected. In addition, the MAC layer of the early Motes was not very sophisticated. The AM receiver transferred the noise as well as the digital Mote communications to the microcontroller, which would regularly lock-up for no "apparent" reason. We believe that the use of the AM transceiver led to many of the Motes not responding during tests, as well as sharp glitches in the recorded data.

\subsubsection{Experimental Data}

Of the 20 experimental wireless sensor nodes used in this experiment, nine motes gave useful data. From these nine data streams, one was disrupted halfway through the blasting sequence (sensor node \#23) and one started to record about onethird of the way through the experiment (sensor node \#22). Two axes of acceleration were collected from each node, with the full plots found in Mitrani et al., (2002). Two Motes (\#24, \#33) were placed within a meter of PARI sensors (\#AC4, \#AB3), and a comparison of the outputs is a good test of Mote performance. The comparison of the X-direction of UCB 33 and PARI AB3 is shown in Fig. 2. The data from each device was band-pass filtered to $0.5-10 \mathrm{~Hz}$ and resampled at 40 samples per second and aligned in time by cross-correlation analysis. The correlation coefficient of the two signals is 0.78 in both cases (UCB sensor \#24 vs. PARI sensor AC4, and UCB sensor 33 compared vs. PARI sensor AB3). The time domain comparison is not as good as for the wood building test, possibly due to the difference in mounting the two different sensors, as seen in Fig. 2. The PARI device was mounted into the ground while the UCB device sat up off the ground about $125 \mathrm{~mm}$ on an aluminum platform which could act as a resonator. It is also possible that the signal was different over the one meter separation. Figure 2 also shows the estimated frequency response for the two (UCB and PARI) time signals. The calculation was made with a 6,6,1 ARX estimation, and it is seen that the results are the same within the expected uncertainty.

\subsubsection{Results From Tokachi}

The experimental motes worked well, considering they were not specifically designed for use in an extreme environment, such as a controlled blasting experiment in winter. This experiment provided much insight into the inadequacies of the Motes. Both the motherboard and the secondary sensor board are dependent on a consistent power source. It is known that battery operation is temperature dependent; the cold, wet weather of the Port of Tokachi may have played a role in the motes behaving inconsistently. Also, these experimental Motes were still susceptible to corruption due to frequencies of similar bandwidth. From this and subsequent experiments at UC Berkeley, we conclude that the Mote behavior worsens while their antennas are parallel to the ground (horizontal position). This explains the relative success of motes in the improved ground zone, where all antennas were in a vertical position, to the ones in PARI's study area. Simple packaging changes, such as a tougher exterior for experiments where harsh conditions are expected, can drastically improve the utility of these devices. 

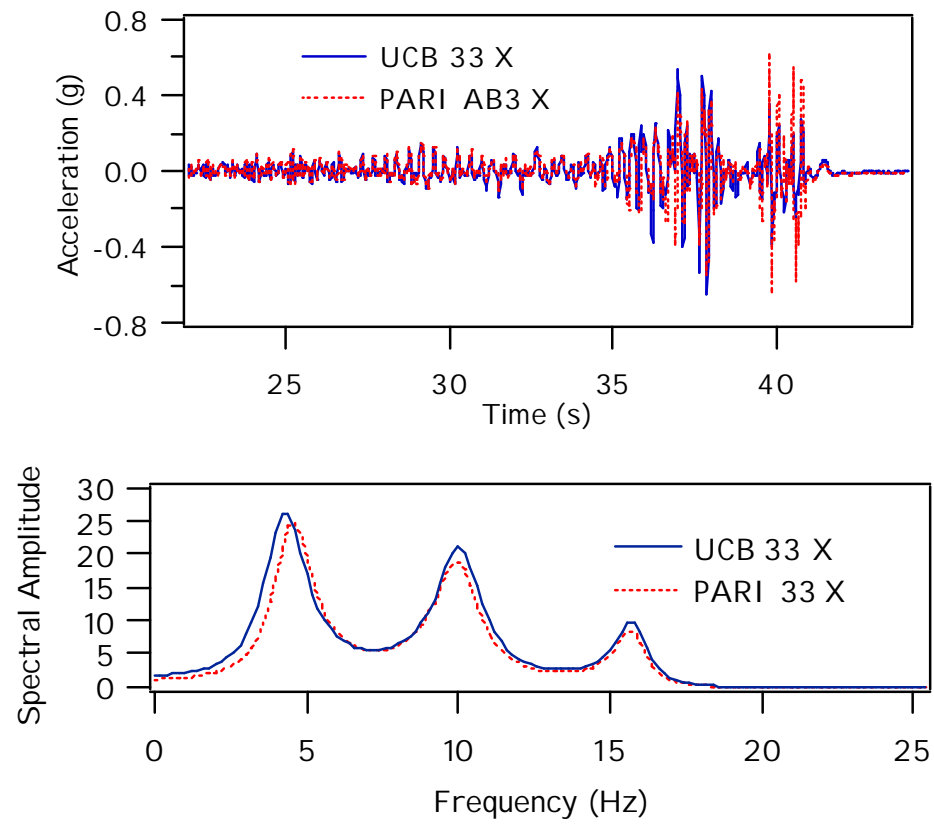

Figure 2. Comparison of the time history recorded by UCB Mote 33 ant PARI accelerometer AB3. The ARX-based spectral estimate for the two are compared in the lower figure.

\subsection{Wood-Frame Building}

The wood-frame campaign was a piggy-back on the CUREe-Caltech Tuck-Under Parking Apartment Building experiment performed by Profs. Mosalam (http://www.ce.berkeley.edu/ mosalam/) and Mahin. A number of such buildings experienced collapse, near collapse, or severe damage in the 1994 Northridge Earthquake. The project consists of dynamic experimental evaluation of a full-scale structure subjected to full 3-D earthquake motions on the Richmond Field Station shake table. A view of the ground floor of the structure, as it sat on the extended shake table, is shown in Fig. 3. The structure was normally instrumented in the traditional manner, resulting in the set of wires shown in Fig. 4. Additional instrumentation was provided by Caltech, which placed a geophone (velocity sensor) on the ground floor and on the top floor, to provide input for their global earthquake damage assessment method (Porter el al., 2002). We installed twenty five 2-D accelerometer Motes on the bottom floor, east-side wall, and thirty three on the main glu-lam beam, as shown in Fig. 3. Most of the Motes were donated by Marathon Products, and were potted on a small sheet of $6 \mathrm{~mm}$ thick plywood so that they could be easily screwed into the structure. The later Motes were supplied by Crossbow in a weatherproof container.

It quickly became apparent that radio communications were much better during physically quite times. Large numbers of people alone were enough to upset communications, and the use of cell phones, radios, television cameras, and other electronic gear further disrupted wireless communications. The problem again was "unexplained" locking-up of the Mote microcontroller, a problem at Tokachi Port, and in all honesty, everywhere the RFM-radio Motes were used. The campaign consisted of about twenty strong shakes over the month of December, 2001. This resulted in the recording of a tremendous volume of data - two minutes of signal from 116 accelerometer channels sampled 100 times per second. Part of the utility of the Motes was lost due to the problems in communications - each Mote had to be down loaded separately with the lap-top base station close by. However, this campaign was probably the largest scale dense instrumentation monitoring ever..

The benefit of the Mote ideal for the dense-pak ${ }^{\mathrm{TM}}$ approach is nicely illustrated by some of the results of the test series. The only visible damage to the structure through all the shaking was some cracking of the stucco at the bottom north 


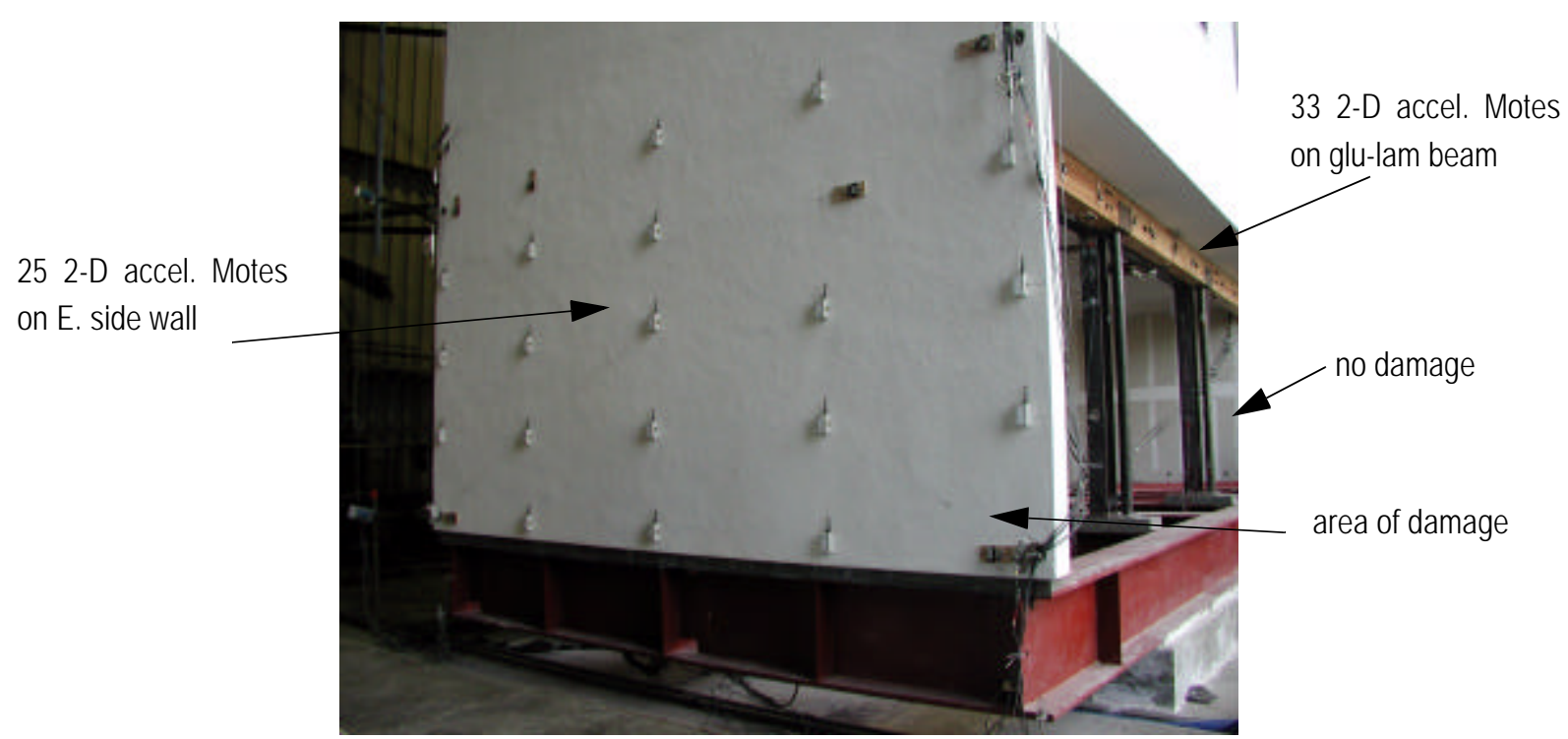

Figure 3. First dense-Pak ${ }^{\mathrm{TM}}$ instrumentation using wireless Motes on building structure. Instrumentation by Glaser on full-scale shake table test by Prof. K. Mosalam, 12/20/01.

corner of the east wall (marked in Fig. 3). Figure 5 shows an FEM analysis performed by Prof. Mosalam (UCB) of the structure subjected to $86 \%$ of the Canoga Park temblor. Note that there is a stress concentration on the east wall, and a stress minimum at the same area on the west wall. This might not be expected since the structure is symmetric, but in fact the 3-D loading is not symmetric! The pair of velocity sensors used for global analysis would neither see this, nor uniquely identify it if it did. Dense-pak can.

There has been some concern about the ability of the inexpensive ADXL202 accelerometers to accurately quantify the structural vibrations. In order to come to some closure on this question, we placed a couple of our Motes directly alongside the Wilcoxen devices traditionally used. The left side of Fig. 6 shows a comparison of the outputs of the two sensors
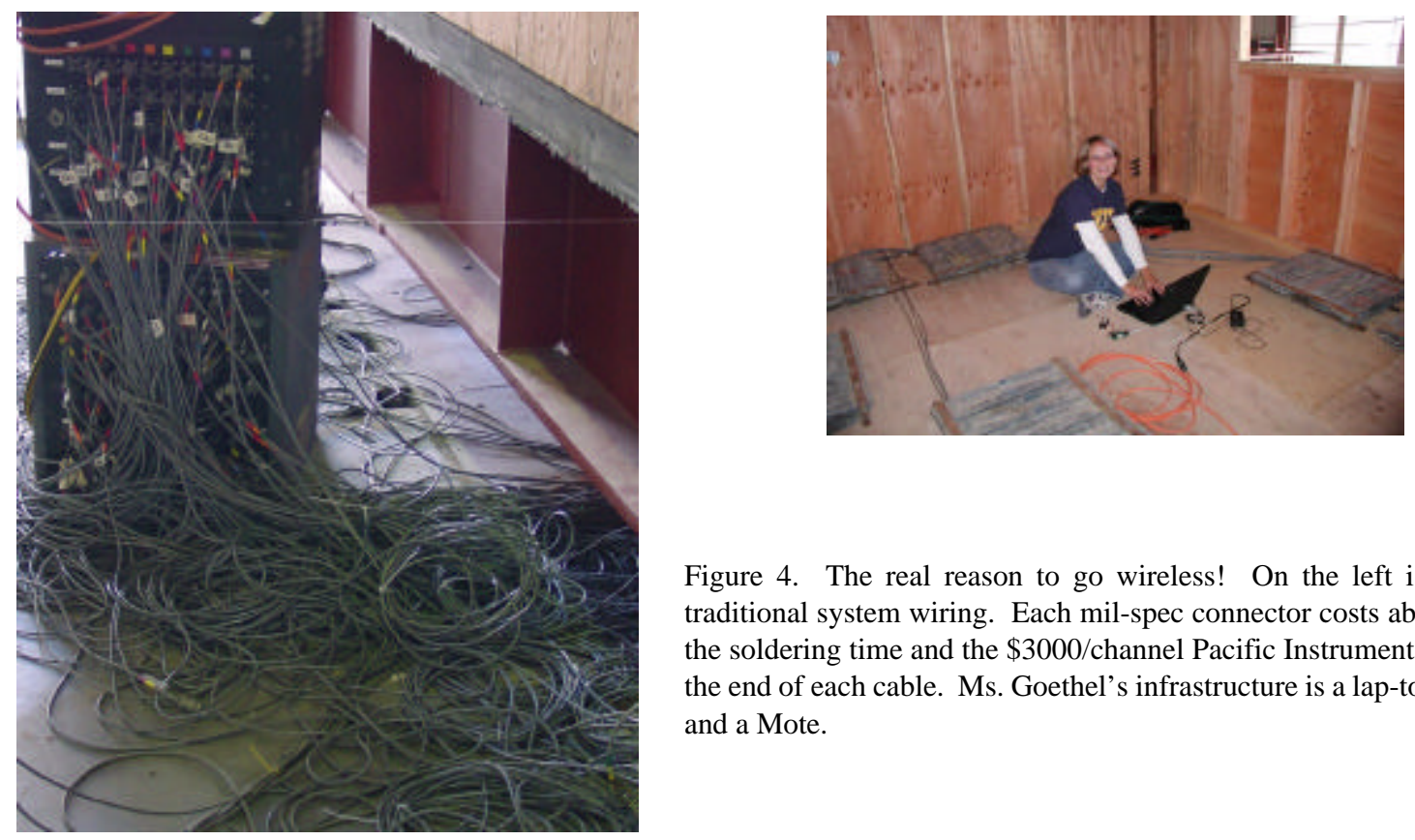

Figure 4. The real reason to go wireless! On the left is part of the traditional system wiring. Each mil-spec connector costs about $\$ 35$, plus the soldering time and the $\$ 3000 /$ channel Pacific Instruments amplifier at the end of each cable. Ms. Goethel's infrastructure is a lap-top compouter and a Mote. 

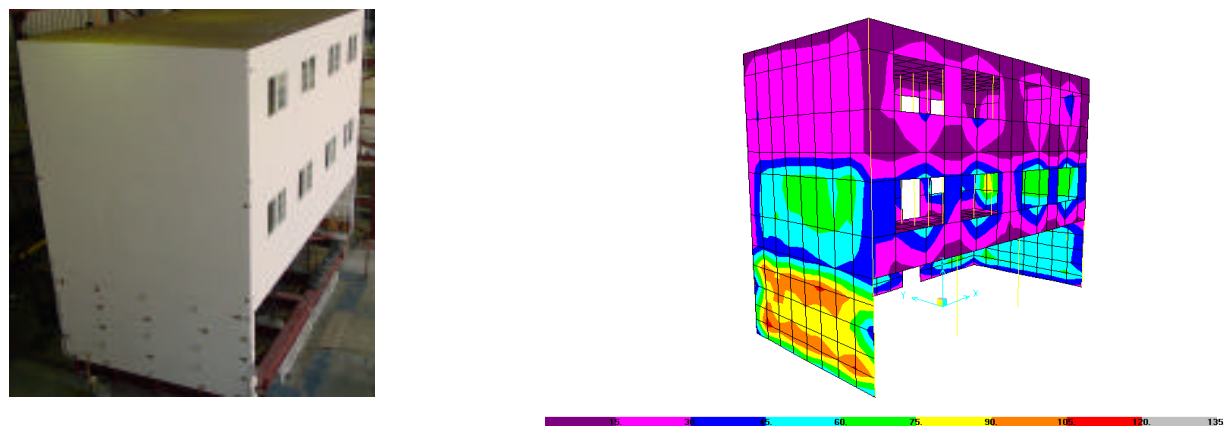

Figure 5. Contour plot of the shear stresses in units of psi for 3-component shaking - the Canoga Park temblor scaled to $86 \%$ of original. (courtesy of Prof. K. Mosalam)

during one of the shakes. Both traces are there, and are identical. The right side shows the spectral estimate for the two, and again we see that they are functionally the same.

\section{DISCUSSION AND COMMENTS}

There have been significant improvements in the Mica (Hill and Culler, 2002) Crossbow hardware and TinyOS over the Rene devices we used for the above discussed case studies. In particular, the RFM transceiver has been replaced by a first-rate unit from Chipcon, which eliminates the unexplained lock-ups, vastly extends battery life, and reliably extends the range to about 100 meters. The author has run tests in Japan, observing the active frequency spectra with an RF spectrum analyzer. Even with twenty people using their handy phones, the Chipcon tranceived data reliably and without interference. Power control circuitry has been added and the microcontroller updated to a more modern version of the 8bit Atmel. The present Crossbow Mica Mote, however, is still basically the device developed and used by the Culler group and the Intel at Berkeley laboratory. The hardware is not electronically robust. A recent discussion on the TOS list serves to illustrate: "The RS-232 level converter that's present on these boards can generate noise that the CC1000

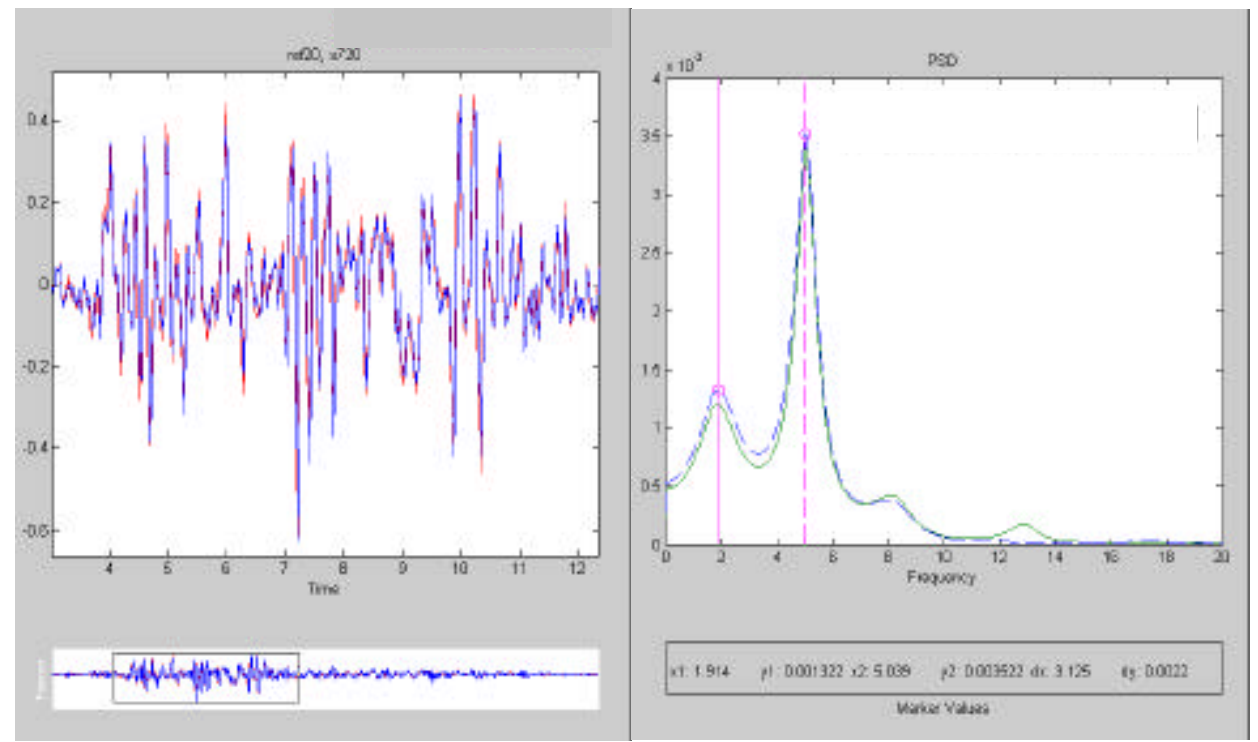

Figure 6. Comparison of the respose of the traditional Wilcoxen piezoelectric accelerometer and the ADXL202e to a strong motion event. Both traces are there, but they are identical. The right plot shows the ARX $(6,6,1)$ estimate of the spectral content of the shaking. 
(especially the $916 \mathrm{MHz}$ configurations) are very susceptible to. A solution that seems to work is that if you put batteries in the mica2"; "Nice work around! I will try it."

The device is an experimental platform for computer science students to learn about developing and implementing advanced wireless sensor webs. Towards this goal, the Motes and TinyOS have been a wild success. This is what the initial development was for, and it was only people such as the author who decided to use them as hardened and developed hardware. As a robust reliable tool for gathering experimental data, we must conclude that there still are many problems in using the Mote devices available from vendors such as Crossbow Inc. These devices are in practice solesource packages, and the user is beholden to the manufacturer to make them work properly. The experiances of relatively competent Mote users (e.g. Berkeley, LBNL, UCSD, LANL, MIT, Senera Inc., Shinkawa Sensor Technologies, etc.) have shown that the devices most often do not work as advertised. It is our opinion that a scientist or engineer who wants to use wireless sensor node technology should not have to earn a Computer Science Ph.D. in order to do so.

TinyOS-based devices are attempting to do too tmanyhings at once, and are not fully successful at any. The Smart Dust concept, of which Motes and TinyOS are the foundation, is attempting optimization of too many variables to allow it to be any where near robust enough for the engineering user. In actuality, any user of wireless sensor networks will only use a small subset of system capabilities at any one time; current multi-hop routing protocols are based in large part on the number of dropped data packets, which is acceptable if the data being recorded and transmitted is not volatile. For example, ambient temperature changes slowly so if a transmission of temperature at time $t$ is lost, the temperature can be retaken and retransmitted at time $\boldsymbol{t}+\mathbf{2 0} \mathbf{m s}$ with no negative effect. If the data is a time series from an earthquake event that happens only once, that data must not be lost.

The commercial devices offer a large non-volatile memory buffer based on EPROM memory, but NesC and TinyOS code does not fully support this memory. In addition there are serious technical problems with the type of memory chip being used. The device is made for long-term storage of very infrequently changed data. The chip has a limited number of write cycles, is slow to write to and read from since internal capacitor banks must be charged for each write. These capacitors use large pulses of power that often "rattles" the Mote microcontroller.

The early devices from Dust Inc., while based on a much more robust MSP430 microcontroller hardware platform, suffered from using what was ultimately a TinyOS-based operating system. These devices could not reliably access flash memory for buffering of recorded data. There were also problems with multi-hop data transmission capabilities and long term operation. This led to our cancelling an environmental monitoring campaign for the Mogau Grotto, Dunhuang, China. The new Dust Inc. Device is based on a completely new, much simplified operating system limiting the scope of the Mote - it now serves to move data and does not look at performing instrumentation and data acquisition tasks. The hardware has been improved by replacing the EEPROM with proper SRAM.

In fact, advanced processing of complex sensor data is the space from which commercial developers are moving away. Ember Inc. calls itself "experts in embedded wireless networking," offering customers low-cost, low-power, and high performance best-in breed radio chips, embedded networking software optimized for control applications, and straightforward evaluation, development, and network management tools and services. The business plan of Dust Inc. has moved towards the data streaming model, providing the link for simple data such as thermostats or security. The science/ engineering user is not a market driver and will always be left out.

\section{CONCLUSIONS}

In this paper we have presented two case histories of the use of wireless Mote technologies. These are devices that incorporate communications, processing, sensors, sensor fusion, and power source into a package currently about two cubic inches in size. They are networked autonomous sensor nodes designed to be embeddable throughout the environment - low cost (\$200 each now, \$5 each in the near future), non-obtrusive, unattended or unmanaged, and dynamically reprogrammable. The first case discussed is the November, 2001, instrumentation of a blast-induced 
liquefaction test in Tokachi Port, Japan. The second case discussed is the dense-pak ${ }^{\mathrm{TM}}$ instrumentation of the seismic shaking test of a full-scale wood-frame building on the UCB Richmond shake table. This paper shows the utility of dense instrumentation, and how it can allow the user to locate damage globally unseen. A methodology of interpreting structural seismic response by Bayesian updating and Kalman filtering was also presented. It is shown that dense, inexpensive instrumentation is needed to identify structural damage and prognosticate future behavior.

The case studies show that while current families of Motes are very useful and are a window to the future, the hardware still has difficulties in terms of reliability and consistency. Features such as memory buffering do not work properly. It also becomes apparent that the TinyOS is a wonderful tool for computer science education but is not an industrial quality instrumentation system for the user. These are, of course, growing pains of the first incarnations of Berkeley's Smart Dust ideal. We expect the dream of easy to use, inexpensive, smart, wireless sensor networks to become a reality in the next couple of years.

\section{ACKNOWLEDGEMENTS}

There are many people and organizations we would like to thank. It is absolutely true that none of this could have been done without them. Dr. Cliff Astill at NSF believed in our work and saw that Nick Sitar and I received an SGER grant (CMS-0126743). Crossbow Inc., particularly Alan Broad, worked extremely hard to see that the devices for Tokachi and the wood-frame test worked. Prof. David Culler, and the Intel at Berkeley laboratory (especially the wonderful graduate students) were key to our success (such as it is). Robert Szewczyk and Jason Hill deserve much of the glory. Marathon Products Inc., built the first 50 Motes for us. Professor Khalid Mosalam was masterful with the wood frame project, which he ran. Prof. Nick Sitar was a key part of all work described here. Special thanks to Sugano-san at the Port and Airport Research Institute, Japan. Finally, the field work was carried out by Judy Mitrani and Jan Goethels, who started from scratch in the summer of 2001, and achieved the impossible.

\section{REFERENCES}

Arici, Y., and Mosalam, K.M., (2000) System Identification and Modeling of Bridge Systems for Assessing Current Design Procedures, Proceedings of SMIP2000 Seminar.

Baise, L.G., and Glaser, S.D., (2000), Repeatability of Site Response Estimates Made Using System Identification, Bulletin of the Seismological Society of America, 90(4), 993-1009.

Beck, J.L., (1978), Determining Models of Structures from Earthquake Records. Earthquake Engineering Research Laboratory 78-01. California Institute of Technology.

Beck, J.L., and Katafygiotis, L.S., (1998), Updating Models and Their Uncertainties. I: Bayesian Statistical Framework, Journal of Engineering Mechanics, 124(4), 455.

Buonadonna, P., and Hill, J., (2000), Active Message Communication for Network Attached Devices, Infocom 2000, Twentieth Annual Joint Conference of the IEEE Computer and Communications Societies, April 22 - 26, 2001

Ching, J.-Y., and Glaser, S.D., (2003), Identification of Soil Degradation During Earthquake Excitations by Bayesian Inference, Earthquake Engineering and Structural Dynamics, 32, 845-869.

DeHoop, M., and DeHoop, A.T., (2000), Wavefield Reciprocity and Optimization in Remote Sensing, Proc. R. Soc. Lond. A, 456(2000) 641-682.

DesRoches, R., Fenves, G.L., (1994), Response of the Northwest Connector in the Landers and Big Bear Earthquakes, Report No. UCB/EERC-94/12, Earthquake Engineering Research Center, University of California, Berkeley.

Farrar, C.R., and Doebling, S.W., (1997), Lessons Learned from Applications of Vibration-Based Damage Identification Methods to Large Bridge Structures, Proc. of the International Workshop on Structural Health Monitoring, Stanford, CA, Sept 1997, 351-370. 
Farrar, C.R., Doebling, S.W., and Nix, D.A., (2001), Vibration-Based Structural Damage Identification, Philosophical Transactions of the Royal Society: Mathematical, Physical \& Engineering Sciences, 359(1778), 131 - 149.

Gersch, W., and Brotherton, T., (1982), Estimation of Stationary Structural System Parameters from Non-stationary Random Vibration Data: a Locally Stationary Model Method, J. Sound and Vibration, 81(2), 215-227.

Glaser, S.D., and Baise, L.G.., (2000), System Identification Estimation of Damping and Modal Frequencies at the Lotung Site, Soil Dynamics and Earthquake Engineering, 19(6), 521-531.

Hill, J., and Culler, D., (2002), Mica: A Wireless Platform for Deeply Embedded Networks, IEEE Micro., 22(6), 12-24.

Hill, J., Szewczyk, R., Woo, A., Hollar, S., Culler, D., and Pister, K.S.J., (2000), System Architecture Directions for Networked Sensors, The Ninth International Conference on Architectural Support for Programming Languages and Operating Systems, Nov. 13-15, 2000.

Hoshiya, M., and Saito, E., (1984), Structural Identification by Extended Kalman Filter, J. Engng. Mech., 110(12), 1757.

Lin, J.-S., and Zhang, Y., (1994), Nonlinear Structural Identification Using Extended Kalman Filters, Computers and Structures, 52(4), 757.

Lus, H., Betti, R., and Longman, R.W., (1999), Identification of Linear Structural Systems Using Earthquake Induced Vibration Data, Earthquake Engineering and Structural Dynamics, 28, 1449-1467.

Mainwaring, A.M., and Culler, D.E., (1999), Design Challenges of Virtual Networks: Fast, General-Purpose Communication, Proc., 1999 ACM Sigplan Symposium on Principles and Practice of Parallel Programming (PPoPP‘99), 34(8), ACM Sigplan Notices, 119-130.

Mitrani, J., Goethals, J., and Glaser, S.D., (2002), Field Testing of Wireless Interactive Sensor Nodes, report submitted to Port and Airport Research Institute, February 13, 2002

McConnell, K.G, (1995), Vibration Testing: theory and practice, Wiley, p. 606.

Porter, K.A., Beck, J.L., Seligson, H.A., Scawthorn, C.R., Tobin, L.T., Young, R., and Boyd, T., (2002), Improving Loss Estimation for Woodframe Buildings, Vol. 1, Report, Consortium of Universities for Research in Earthquake Engineering, Richmond, CA, pp. 136.

Safak, E., (1997), Propagation of Seismic Waves in Tall Buildings, Tall Buildings for the 21st Century (Proceedings of the 4th Conference on Tall Buildings in Seismic Regions), 129-154.

Safak, E., (1988), Analysis of Recordings in Structural Engineering: Adaptive Filtering, Prediction, and Control. (OpenFile Report 88-647). Menlo Park, CA: U.S. Geological Survey.

Shumway, R.H., and Stoffer, D.S., (1982), An Approach to Time Series Smoothing and Forecasting Using the EM Algorithm, Journal of Time Series Analysis, 3(4), 253.

Smyth, A.W., Masri, S.F., Chassiakos, A.G., and Caughey, T.K., (1999), On-Line Parameter Identification of MDOF Nonlinear Hysteretic Systems, Journal of Engineering Mechanics, 125(2), 133.

Stewart, J.P., Fenves, G.L., (1998), System Identification for Evaluation of Soil-sStructure Interact Effects in Buildings from Strong Motion Recordings, Earthquake Engineering and Structural Dynamics, 27, 869-885.

TOS, (2004), http://webs.cs.berkeley.edu/tos/index.html.

Udwadia, F. E., (1985), Some Uniqueness Results Related to Soil and Building Structural Identification, SIAM J. Applied Math., 45(4), 674.

Werner, S.D., Crouse, C.B., Katafygiotis, L.S., and Beck, J.L., (1994), Use of strong motion records for model evaluation and seismic analysis of a bridge structure, Proc. Fifth U.S. National Conference on Earthquake Engineering, 1, 511-520.

Woo, A., and Culler, D., (2003), Evaluation of Efficient Link Reliability Estimators for Low-Power Wireless Networks. Technical Report UCB//CSD-03-1270, U.C. Berkeley Computer Science Division, September 2003. 